Monatsschrift f. Geburtshülfe u. Gynäkologie 1924;67:247-252

\title{
V. Literaturverzeichnis
}

\section{Geburtshülfe.}

Andrews, Fall von verhaltener Plazenta. J. Amer. med. Ass. 31. V. 24.S. 1780. Bauer, Gibt es eine konstitutionelle Veranlagung zur Zeugung von Nach-

kommen vorzugsweise eines Geschlechtes? Klin. Woch. Nr. 21. S. 928. Baamrn, Zur

künstlichen Atmung bei Asphyxia neonat. Dtsch. med.

Woch. Nr. 20. S. 645. - Bemerkung zum Aufsatz von J. Amreich: Zur Ätiologiß der von den Uterusvenen ausgehenden Luftembolie. Z. f. G. Nr. 22. S. 1201. de Bíasi, Chinin,

Schwangerschaft und Malaria. La Clinica. Juni 1924

Nr. 6. S. 223.

248

Literaturverzeichnis.

Broßmann, Sectio caesarea bei Atresia vag. cong. Ztschr. f. Gyn. Nr. 23.

S. 1262. Brack, Sßmmelweiß. Munch, med. Woch. Nr. 20. S. 645. v. Büben, Die Rolle des Koitus bei vorzeitigem Blasensprung und Er-

krankungen des Kindbetts. Z. f. G. Nr. 24. S. 1310. Burger, Die Behandlung der

Nachgeburtsperiode nach Baers Verfahren.

Ztschr. f. Gyn. Nr. 20. S. 1082. Eberhart, Zur Fortpflanzungsregulierung. Z. f. G. Nr. 21. S.

1155. Fink, Akute transitorische Erblindung post partum. Ztschr. f. Gyn.

Nr. 22. S. 1188. Freund und Berger, Über Befunde von Streptokokken im Blute. Dtsch.

med. Woch. Nr. 20. S. 625. Greetlhill, Vorzeitige Ablösung der Plazenta durch die

Kiellandzange.

Z. f. G. Nr. 23. S. 1258. Haselhorst und Papendieck, Hämatin als physiologischer Bestandteil desBlutes

inder FötalperiodeundbeimNeugebornenen. Klin. Woch. Nr. 22. S. 979. Heidler, Beitrag zur

Bedeutung der ruhenden Infektion für die Geburts-

hilfe nebst Bemerkungen über deziduale Veränderung in Polypen der

Portio. Arch. f. Gyn. Bd. 121. S. 429. Hoffmann, Eklampsie und Epilepsie, kasuistischerBeitrag. Z. f. G.

Nr. 22. S. 1184. Holzbach, Die Wiederbelebung asphykt. Neugeborener mit Sauerstoff.

Z. f. G. Nr. 23. S. 1256. Hülse, Zum Eklampsieproblem. Z. f. G. Nr. 22. S. 1179. Kaboth, Über die Wirkung eiweißreicher Nahrung auf den schwangeren

Organismus mit besonderer Berücksichtigung der Harnstoffbildung.

Arch. f. Gyn. Bd. 121. S. 631. Kemper, Über die terminale Gewichtsabnahme Schwangerer.

Arch. f. Gyn.

Bd. 121. S. 604. Knebel, Riesenovarialkystom am Ende einer gleichzeitig bestehenden

Schwangerschaft. Z. f. G. Nr. 20. S. 1079. Koerting, Gynergen zur Bekämpfung der Atonia uteri (Bemerkungen zu

der Arbeit von Sophie Lützenkirchen in Nr. 49 der Munch, med. Woch.). 
Munch, med. Woch. Nr. 23. S. 753. Kok, Schwangerschaft und Karzinom. Z. f. G. Nr. 21. S. 1136. Kiírthy, Die chemischen Veränderungen des Blutes in einem Falle von akuter hämorrh. Schwangerschaftsnephritis. Munch, med. Woch.

Nr. 22. S. 709. Langstein, Mästung von Säuglingen. Dtsch. med. Woch. Nr. 20. S. 638. Lederer, Der Einfluß der Schwangerschaft auf das Wachsen und Rezidi-

vieren maligner Geschwülste. Z. f. G. Nr. 24. S. 1289. Lewis, Hofbauerzellen (Clasmatocyten) der menschlichen Chorionzotten.

Bull. John Hopkins Hosp. Juni 1924. S. 183. Lüttge, Junge oder Mädchen ? Serologische Geschlechtsbestimmung des

Kindes im Mutterleibe. Z. f. G. Nr. 21. S. 1139. Mahnert, Untersuchungen über Veränderungen des Stoffwechsels und des

Körpergewichtes in der Schwangerschaft. Arch. f. Gyn. Bd. 121. S. 620. Meyer, Medikamentöse Behandlung des Herzgefäßsystems in der Chirurgie.

Klin. Woch. Nr. 22. S. 988.

Literaturverzeichnis. 249

v. Míkulicz-Radecki und Lueg, Über den Einfluß der Aortenkompression auf die Spontanbewegungen des Kaninchenuterus. Z. f. G. Nr. 21.

S. 1132. Mueller, Die Mechanik der Geburt. Arch. f. Gyn. Bd. 121. S. 502. Nothmann, Über Schwangerschaftsglykosurie. Klin. Woch. Nr. 23.

S. 1019. Pinkus, Über den Schutz vor Syphilisinfektion durch interne Medikamente. Med. Klin. Nr. 22. S. 739. Ruge, Studien zur Virulenzprüfung der Streptokokken. Arch. f. Gyn. Bd. 121. S. 363. Simon und Wellewa, Icterus neonat. •und Widalsche Reaktion. Z. f. G. Nr. 23. S. 1250. Sommer, Die septischen Allgemeininfektionen, ihre Prognose und Beurteilung medikamentöser Behandlung durch das Hämogramm.

Z. f. G. Nr. 21. S. 1143. Schulie, Die Stellung des Kaiserschnittes in der Behandlung der Placenta praevia. Z. f. G. Nr. 20. S. 1065. Schwarz, Über Alter und erste Konzeption der Frau. Z. f. G. Nr. 24.

S. 1282. Schwarzkopf, Sub partu perforiertes Pyovarium. Z. f. G. Nr. 24. S. 1303. V. d. Steinen, Untertemperaturen bei gesunden Neugeboreneh. Z. f. G.

Nr. 23. S. 1254. Stern und Schwarz, Klinisches zum Geburtentrauma. Klin. Woch. Nr. 21.

S. 931. Vogt, Zur Kritik der Uterus-Scheidentamponade. Z. f. G. Nr. 22. S. 1195. Volkmann, Ererbter Milchmangel bei guter Ausbildung der Brust. Z. f. G.

Nr. 22. S. 1198. Wagner, Zum transperit. Kaiserschnitt bei Eklampsie. Z. f. G. Nr. 23.

S. 1263. Westphal, Akutes Lungenödem in der Schwangerschaft. Z. f. G. Nr. 22.

S. 1191. Young, Vorläufiger Bericht über einen Fall von gemischtem Geschlecht:

anscheinend männlich mit einem Hoden im Skrotum rechterseits,

Eierstock, Tube und Uterus im Leistenkanal links. Bull. John

Hopkins Hosp. Juni 1924. S. 165.

Gynäkologie.

Bauer und Wehefritz, Gibt es eine Hämophilie beim Weibe ? Arch, f. Gyn.

Bd. 121. Г. 462. Bergl, Ein neues Okklusivpessar. Dtsch. med. Woch. Nr. 23. S. 763. Bompiani, Seheidenzerreißung beim Koitus. La Clinica ostetr. Juni 1924.

Nr. 6. S. 230. Boeters, Die operative Unfruchtbarmachung der Blödsinnigen, Geistes-

kranken usw., gewürdigt von der rechtlichen Seite (Erwiderung auf

den Aufsatz S. 443 der Munch, med. Woch.). Munch, med. Woch. 
Nr. 21. S. 685.

Bonne, Zur Verhütung und Behandlung des Dekubitus. Med. Klin. 23.

S. 784.

250

Literaturverzeichnis.

Bröse, Der Flour genitalis der Virgines. Med. Klin. Nr. 21. S. 703. Brüning, Über die

Desinfektion der chirurg. schneidenden Instrumente,

insbesondere über die Unzuverlässigkeit des Alkohols. Dtsch. med.

Woch. Nr. 22. S. 717. Budde, Über die Entstehung der Keratome. Klin. Woch. Nr. 21. S. 942.

Cowburn, Blutdruck bei Operationen und allgemeine Anästhesie. J. Amer.

med. Ass. V. 31. S. 1748. Cra $c$ ce und Knickerbocker, Primäres Ureter-Karzi $\pi$ öm (Epitheliom).

J. Amer. med. Ass. 14. VI. S. 1930. Davis, Kellay, Amolsch, Anatomische und klinische Studien über 875 Pla-

zenten. Amer. J. obst. gyn. VII. 6. S. 637. Dietrich, Zum Fruktulet. Munch, med. Woch. Nr. 20.

S. 666. Ebermayer, Rechtsfragen aus der ärztlichen Praxis (Abschaffung der

$\S \S 218$ und 175 Str.G.B., ärztliches Berufsgeheimnis, Vernichtung

lebensunwerten Lebens). Dtsch. med. Woch. Nr. 24. S. 806. Gaifami, Utèrussyphilis. Clinica

ostet. Juli 1924. Nr. 6. S. 209. Gal, Pseudohermaphroditismus feminin. bei 2 Schwestern. Z. f. G.

Nr. 22,

S. 1203. Haberlandt, Erwiderung auf A. Greils „Einwände gegen die hortnonale

Sterilisierung nach Haberlandt”. Z. f. G. Nr. 22. S. 1197. Halban-Seiíz, Biologic und Pathologie

des Weibes. Liefg. 8. Stickel, Vor-

und Nachbehandlung gynäkologischer Operationen. - Thaler, All-

gemeinnarkose und Lokalanästhesie in Geburtshilfe und Gynäkologie. Hall, Großes interstitielles Zervixfibrom. Operationen. Am. J. obstet.

gyn. VII. .6. S. 709. Hellendall, Lebenrettende Wirkung durch Lobelin. hydrochlor. crist. bei schwerer Oligopnoe in Pantopon-Scopolamin-Äthernarkose. Z. f. G.

Nr. 21. S. 1151. Hirsch, Die Dysmenorrhoe der Spasmophilen und über die Ursachen des

Menstruationsschmerzes. Z. f. G. Nr. 20. S. 1073. Hoffmann, Fall von Uterusstein. Z. f. G. Nr. 24. S. 1305. Jaffé, Bau und Funktion des Corpus luteum. Z. f. G. Nr. 21. S. 1122. V. Jaschke, Zur Symptomatologie der Retroversioflexio uteri. Munch.

med. Woch. Nr. 21. S. 667. Jüngling, Das Mammakarzinom und seine zeitgemäße

"strahlentherapeu.

tische und chirurgische Behandlung. Bemerkung z $\backslash ı$ der Arbeit von

H. Jarre in Nr. 3 der Klin. Woch. S. 584. Klin. Woch. Nr. 24. S. 1077. Kahn, Die

Differentialdiagnose maligner Tumoren aus wenigen Tropfen

Blut. Klin. Woch. Nr. 21. S. 920. Küstner, Beitrag zur Spontanamputation der Adnexe. Z. f. G.

Nr. 24

S. 1294. Levy, Die Enteroptose. Klin. Woch. Nr. 21. S. 938. Liepmann und Brusten,

Sammelreferat aus dem gesamten Gebiet der.

Gynäkologie. Med. Klin. Nr. 22. S. 755.

Die Psychologie in der Fre, uenheilkunde. Z. f. G. Nr. 21. S. 1139.

Zur Ätiologie der Metropathia uteri. Munch, med. Woch. Nr. 21. S. 671. Lütlge,

Lebenbedrohliche Ovarialblutungen. Z. f. G. Nr. 24. S. 1297.

Literàturverzeichnis. 251

Lynch, Eine neue Operation wegen Mastdarmvorfall. Vorläufiger Bericht. 
J. amer. med. Ass. 14. Juni 1924. S. 1930. Lunz, Erfahrungen mit „Dicodid”, einem der Morphium- und Kodeingruppe nahestehenden neuzeitlichen Präparat· Z. f. G. Nr. 22.

S. 1202. Mandelstamm, Zur Symptomatologie des äußeren Fruchtkapselaufbruchs. mit frischem Bluterguß in die Bauchhöhle. Z. f. G. Nr. 23. S. 1260. v. Oettingen, Die Entstehung der Schokoladenzysten aus heterotopen

Epithelwucherungen des Ovars. Z. f. G. Nr. 21. S. 1129. Oldag, Über Verhaltung von Sk $\beta$ letttrümmern menschlicher Föten in der Gebärmutter (intrauterine Skelettierung). Dtsch. med. Woch. Nr. 22.

S. 716. Opitz, Welche Vorstellungen sind heute über Entstehung und Heilbarkeit des Krebses erlaubt? Munch, med. Woch. Nr. 21. S. 668. Perazzi, Verkannte vollentwickelte Extrauterinschwangerschaft. La clinica

ostet. Juni 1924. Nr. 6. S. 232. Pestalozza, Amenorrhoe bei Genitaltuberkulose. La clinica ostet. Juni

1924. Nr. 6. S. 234. Philipp, Beobachtungen bei der Narcylennarkose. Munch, med. Woch.

Nr. 20. S. 639. Polak und Phelan, Sammelreferat über die Operationen zur dauernden

Erweiterung des knöchernen weiblichen Beckens. Amer. J. obstet

gyn. 6. Juni 1924. S. 739. PuSt, Zur intrauterinen Behandlung (Bemerkungen zu der Arbeit von

Max Nassauer in Nr. 14 der Munch, med. Woch.). Munch, med. Woch.

Nr. 23'. S. 754. Romeo, Steriler Abortversuch im 2.Monat vor 12 Jahren bei Zervikalpolyp.

La clinica ostet. Juni 1924. Nr. 6. S. 228. Seel, Über die Wirksamkeit des Hirtentäschels. Munch, med. Woch.

Nr. 21. S. 676. Singer, Kongenitale Zyste der Niere beim Neugeborenen. Surg. gyn. obst.

Juni 1924. S. 768. Solbach, Die Ungefãhrlichkeit der Narcylenbetäubung. Dtsch. med. Woch.

Nr. 23. S. 739. Scheid und Bukofzer, Über das Verhalten des großen Netzes gegenüber

ektopischen Früchten. Z. f. G. Nr. 21. S. 1141. Schulte, Ergebnisse unserer Behandlung von 536

Genitalkarzinomen aus den

Jahren 1914-1920. Arch. f. Gyn. Bd. 121. S. 446. Thomas, Die strafgesetzlich $\beta$ Natur ärztlicher Eingriffe in dem EntwurE

zu einem deutschen Strafgesetzbuch (Abtrejbung). Med. Klin. Nr. 20,

S. 695 und Nr. 21, S. 730. Viana, Beitrag zum Studium des abdominalen Kaiserschnittes bei

Plac.

praevia. La clinica ostet. Juni 1924. Nr. 6. S. 215. Vogt, Die intravenöse Urotropintherapie der Harnverhaltung. Dtsch. med.

Woch. Nr. 23. S. 737. Warburg, Negelein und Posener, Versuche an überlebendem Karzinomgewebe. Klin. Woch. Nr. 24. S. 1062.

252 Druckfehlerberichtigung. - Personalien und Tagesnachrichten.

Weinzierl, 1st die Vakzinebehandlung der gonorrh. Adnextumoren nur als Proteinkörpertherapie aufzufassen ? Z. f. G. Nr. 23. S. 1244.

- Über Milchbehandlung bei entzündlichen Adnextumoren. Med. Klin-Nr. 23. S. 776.

Wiener, Diabetes mellitus während der Schwangerschaft. Amer. J. obst. gyn. 6. Juni 1924. S. 710 .

Strahlentherapie.

Spieíhoff, Die menorrhag. $\Lambda$ Virkung der Milzbestrahlung. Dtsch. med. Woch. Nr. 23. S. 745.

Druckfehlerberichtigung. 
In der Arbeit von Doedeiiein-Voltz

S. 262 unter der Abbildung Zeile 4 lies Abb. 6 statt Abb. 3, „265 Zeile 5 von unten „, , $8,,, 5$.

Auf Seite 129 dieses Bandes ist unter den Personalien in der letzten Zeile Insterburg anstatt Innsbruck zu lesen.

Personalien und Tagesnachrichten.

Privatdozent Dr. Emil Vogt in Tubingen hat die Dienstbezeichnung außerordentlicher Professor erhalten.

Dr. Heinz Kústner, Assistent der Universitäts-Frauenklinik in Halle, hat sich an der dortigen Universität habilitiert.

Dr. R. Hornung, Oberarzt der Leipziger Universitäts-Frauenklinik, hat sich an der dortigen Universität als Privatdozent habilitiert.

Die Vierteljahrsschrift des Bundes deutscher Ärztinnen, herausgegeben von Dr. Hermine Heusler-Edenhuizen und Dr. Laura Turnau (Verlag C. A. Schwetschk $\theta$ \& Sohn, Berlin) will, wie die Mutter, in der Familie die härtere Art des Vaters ergänzen durch größere Mitarbeit auf Gebieten, die nach ihrer Wesensart der Bearbeitung bedürfen. Im Zusammenschluß der Ärztinnen Deutschlands will sie sozialhygienische Fragen vom Standpunkt der Ärztin als Frau bearbeiten, Vorschläge für die einschlägige Gesetzgebung ausarbeiten, für die nicht mehr arbeitsfähigen älteren Kolleginnen und die jungen Medizinerinnen sorgen, also wissenschaftliche und wirtschaftliche Interessen vertreten. Die erste Nummer wird sehr sympathiser! durch einen Brief der Seniorin Frl. Fr. Tiburtins eingeleitet. L. Rabinowitsch-Kempner behandelt die Frage Ärztinnen und Tuberkulosebekämpfung, M. Wygodzinski spricht Gedanken zur Kassenarztfrage aus. Das Unternehmen entspricht augenscheinlich einem in diesen Kreisen lebhaft empfundenen Bedürfnis. Wir gebea ihm kollegiale Wünsche zu seiner Entwicklung mit auf den Weg! A. Martin.

Der japanische medizinische Verein in Berlin gibt eine japanische medizinische Zeitschrift heraus als Beilage der Nippon Jji Shimpo. Angesichts des überaus regen wissenschaftlichen Austausches der deutschen und japanischen- Forschungsergebnisse begrüßen wir die junge Kollegin mit dem Wunsche, daß es ihr gelingt, diesen Austausch nachhaltig fruchtbar zu gestalten. A. Martin. 